\title{
Our experience of using Hydroxychloroquine and Azithromycin in the treatment of COVID-19: an observational study from Kinshasa, Democratic Republic of Congo
}

\author{
Jean-Marie N. Kayembe ${ }^{1,2 *}$, Daniel Ishoso², Christian Mbongopasi², Don Jethro Mavungu², Jean René Makulo, Stéphane Mukendi², Nsengi \\ Ntamabyaliro $^{1}$, R. Junior Matangila ${ }^{1}$, Jean-Jacques Muyembe ${ }^{1,2}$ \\ ${ }^{1}$ Cliniques Universitaires de Kinshasa,D R Congo.
}

${ }^{2}$ Secrétariat Technique COVID-19, INRB, Kinshasa, D R Congo.

*Corresponding Author: Jean-Marie N. Kayembe, Cliniques Universitaires de Kinshasa, Secrétariat Technique COVID-19, INRB, Kinshasa, D R Congo.

Received date: April 22, 2021; Accepted date: May 04, 2021; Published date: May 07, 2021

Citation: Kayembe JMN, D Ishoso, C Mbongopasi, Don J Mavungu, Jean R Makulo. (2021) Our experience of using Hydroxychloroquine and Azithromycin in the treatment of COVID-19: an observational study from Kinshasa, Democratic Republic of Congo. Biomedical Research and Clinical Reviews. 4(1); DOI: 10.31579/2692-9406/065

Copyright: (C) 2021 Jean-Marie N. Kayembe, This is an open-access article distributed under the terms of the Creative Commons Attribution License, which permits unrestricted use, distribution, and reproduction in any medium, provided the original author and source are credited.

\begin{abstract}
Background: Up to now COVID-19 treatment is a matter of great debate. Several trials and observational studies have brought conflicting results regarding the efficacy of some repurposed drugs suggested treating COVID-19, stressing the need for further research.

Objective: This study aimed to add to the existing literature, some evidence on the efficacy and safety of hydroxychloroquine (HCQ) and azithromycin (AZ) as suggested drugs for COVID-19 treatment.

Materials and Methods: This observational study reports the experience of conventional treatment (CT) combining HCQ plus AZ applied in the Democratic Republic of Congo. We compared epidemiological and clinical data between $1,100(89 \%)$ subjects submitted to the CT and 135 (11\%) who did not receive the CT. Survival analyses including KaplanMeier and Cox regression were used to determine the clinical progress at day 10 and the survival likelihood at day 15 , from the treatment initiation date.

Results: Clinical outcome is much better in the CT group on D10 than in the comparison group ( $\mathrm{p}=0.001)$. The adverse effects reported are minor and equivalent in both groups (3\% vs 3\%).

Conclusion: This preliminary observational study suggests a beneficial and safe effect of conventional treatment in our country and justifies the continuation of this therapeutic protocol at this time. However, larger analytical studies with more robust evaluation criteria are needed to strengthen the power of observations.

Keywords: hydroxychloroquine; azithromycin; conventional treatment; COVID-19; Kinshasa; Democratic Republic of Congo
\end{abstract}

\section{Introduction}

Since the first cases of COVID-19 were reported in Wuhan, China, in December 2019, the medical world has faced an unprecedented challenge. Numerous clinical trials of effective drugs against SARS-CoV-2, a wellidentified etiological agent, are regularly published. But despite all these efforts, there is currently no consensual drug optimally effective. Efficacy criteria must incorporate anti-viral and/or immunomodulatory properties of drug candidates currently recommended in clinical trials, and often prescribed compassionately [1].

While randomized clinical studies allow for a better determination of cause-effect relationships between an intervention and outcome, they are not easy to conduct in the face of this pandemic in which the physiopathogenic knowledge of CoV-2 SARS is far from being definitively well known. Researchers undertake observational clinical studies with sometimes questionable power.

They are harnessing knowledge about molecules that have been used for decades in other diseases or against other viruses. Chloroquine belongs to this group of pharmacological agents, known for its anti-viral and immunomodulatory properties [2-5]. With its less toxic derivative, hydroxychloroquine, this drug has been used since the 1950s in the treatment of malaria [6,7], but also in the management of certain inflammatory and autoimmune conditions such as disseminated Lupus erythematosus [8]. Its antiviral properties, demonstrated in vitro, have led many research teams to propose it as a COVID-19 treatment $[9,10]$. 
Previous studies have shown that hydroxychloroquine prevents the virus from entering host cells in two ways. The first, by inhibiting the receptor of the angiotensin conversion enzyme 2 (ACE2). Indeed, the binding of the viral spike protein $(S)$ to the ACE2 receptor is sufficient for serine transmembrane protease (TMPSS2) to induce the cleavage of the $\mathrm{S}$ protein in $\mathrm{S} 1$ and $\mathrm{S} 2$, thus exposing the FP fusion peptide. It is through FP that the membrane of the virus merges with the host cell. This allows the release of viral particles into the cell [7-10].

The other pathway is accomplished, by increasing the $\mathrm{pH}$ (by the input of protons) in the endosomes, reducing the action of the cathepsin- $\mathrm{L}$ and inhibiting the fusion of the viral membrane and endosomes [7-10]. It is possible that other proteases, not influenced by hydroxychloroquine, are also involved in the activation of the viral protein S. However, the drug may also inhibit the release of pro-inflammatory cytokines $(7,11)$. This molecule has been proposed by many teams in pre- or post-exposure prophylaxis, or as a treatment in benign and early-stage forms of COVID19[10-13]. The conclusions of these clinical trials are contradictory. Positive results on symptom reduction, in-hospital time but not mortality have been described in some previous studies $[14,15]$.

Since the first case of COVID-19 reported in Kinshasa, on $10^{\text {th }}$ of March 2020, the Technical Secretariat in charge of the response proposed a monitoring strategy around diagnosis, isolation of all RT-PCR positive cases, and their treatment by the combination of hydroxychloroquine (HCQ)/chloroquine (CQ) - Azithromycin (AZ) - Vitamin C with or without zinc, presented as the conventional treatment. This choice was based on the known antiviral effect of these drugs in vitro and data reported, notably by the IHU of Marseille in France, on the reduction of viral load by $100 \%$ on D7 by this association, as well as on its beneficial clinical outcomes [5].

On the other hand, chloroquine had been extensively used in the treatment of malaria in our country, with an assured safety profile, and is easily accessible, despite the weakness of our health system. Its early use in COVID 19 would have beneficial effects as reported in some clinical trials worldwide [16, 17], in contrast to the negative results reported mainly in clinical trials in North America [18]; however, there is no established evidence of positive action on severe cases and on mortality. The conflicting results of studies on the effectiveness of hydroxychloroquine against COVID-19 have prompted the DRC to choose to consider as much as possible locally generated data as evidence in therapeutic choices.

Current data on the physiopathogenic mechanisms of SARS-COV-2 in COVID-19 have led to a better definition of therapeutic targets for this condition, which may involve multiple organs. The interest in using molecules with anti-inflammatory properties is justified given the existence of a higher inflammatory phase in the disease; substances modulating the production of cytokines are useful for countering the cytokines storm $[19,20]$ and anticoagulants have their indication in this pathology in which exaltation of pro-thrombotic mechanisms has been described [21].

This retrospective study reports observations on analyses of COVID-19 in the hospital. It aims to determine the potential effect of CT on clinical outcomes and survival rate, as well as main adverse events found.

\section{Materials and Method}

We conducted a cohort analysis using routinely collected data from the DRC Ministry of Health's COVID-19 Multi-Sectoral Response Committee database, spanning March 10, 2020-August 31, 2020. All
COVID-19 patients admitted at the ten largest health facilities in Kinshasa were eligible for inclusion. Patients were staged according to the current COVID-19 clinical categories in use elsewhere of mild, moderate, severe, and critical disease. Patients were followed and/or hospitalized at 10 treatment centers in the city of Kinshasa, including: Saint Joseph Hospital $(\mathrm{n}=152)$, Cinquantenaire Hospital $(\mathrm{n}=100)$, HJ Hospital $(\mathrm{n}=52)$, SinoCongolais Hospital $(n=99)$, Kinshasa University Hospital $(n=270)$, Kinshasa Medical Center ( $\mathrm{n}=18)$, Ngaliema Clinic $(\mathrm{n}=347)$, Diamant Clinic ( $\mathrm{n}=34)$, Vijana Medical Center $(\mathrm{n}=189)$, Monkole Medical Center $(n=102)$. The data were collected using an Excel spreadsheet; and analyzed using STATA15 software (StataCorp.2017. Stata Statistical Software: Release 15.College Station, TX: StataCorp LLC)

Treatment and control groups were formed. With 135 patients in the control group out of 1, 235 patients. This second group included patients enrolled before the availability of Chloroquine and those with formal contraindications, including heart conditions such as arrhythmia or elongation of QT interval. Indeed, cardiac toxicity of HCQ has been reported in previous studies $[8,22]$. They were mostly on Artemether/Lumefantrine associated with either Azithromycin or amoxicillin/clavulanic acid.

Sociodemographic, clinical, and paraclinical characteristics were recorded and then compared between the two groups. The proportion for categorical variables, and medians for quantitative as non-symmetrical were used. Statistical tests including the Kruskal-Wallis, Pearson Chi2, Fisher exact, were used as appropriate. The subjects were matched using the nearest neighbor method, and the effects of the treatment were adjusted to the propensity score. The threshold $\alpha-0.05$ has been set to establish the significance of differences observed.

Of the hospitalized patients, 988 were followed up to D15 (arbitrarily determined) to assess their likelihood of survival. The Kaplan-Meier method was used for this purpose and the curves were compared using the Log-rank test. A Cox model was then established, based on an automatic selection of FORWARD-type variables based on the probability ratio (LR), using an entry probability of 0.05 . The measures of association between each independent variable and survival were reported in the form of a Hazard Ratio accompanied by their $95 \%$ confidence intervals. The final model took up only the variables with significant effects after adjustment. Regression was achieved when the verification of the proportionality of hazards was conclusive by a non-significant global test and horizontal-trend Schoenfeld residues, as well as in the presence of parallel in-in curves of predictors over time. The safety of use of conventional treatment was compared to other treatments and evaluated by recording all adverse events (AI) reported by patients and encoded. These were categorized according to version 23.0 of the Medical Dictionary for Regulatory Authorities (MedDRA) and the following parameters were assessed for each AI: severity, duration, time of appearance, actions taken and outcome.

\section{Results}

\section{A1. Effects of conventional treatment}

Of the 1,235 files selected, 1,100 (89\%) were treated by the CT, and 135 (11\%) were assigned to the control group.

\section{Comparison of subject characteristics in both groups}

Table 1 compares some socio-demographic characteristics and does not reveal any significant difference between the groups. 


\begin{tabular}{|c|c|c|c|c|c|c|}
\hline \multirow[b]{3}{*}{ Variables $(\mathrm{n}=1,235)$} & \multirow[b]{3}{*}{$\mathrm{n}$} & \multicolumn{4}{|c|}{ Chloroquine + Azithromycin } & \multirow{3}{*}{ p-value } \\
\hline & & \multicolumn{2}{|c|}{$\begin{array}{l}\text { Yes } \\
(\mathrm{n}=1,100)\end{array}$} & \multicolumn{2}{|c|}{$\begin{array}{l}\text { No } \\
(\mathrm{n}=135)\end{array}$} & \\
\hline & & $\mathrm{n}^{\prime}$ & $\%$ & $\mathrm{n}^{\prime}$ & $\%$ & \\
\hline Suburban residence & & & & & & 0.154 \\
\hline Yes & 291 & 250 & 26.1 & 41 & 32.0 & \\
\hline Not & 795 & 708 & 73.9 & 87 & 68.0 & \\
\hline Sex & & & & & & 0.933 \\
\hline Wife & 427 & 379 & 35.2 & 48 & 35.6 & \\
\hline Man & 785 & 698 & 64.8 & 87 & 64.4 & \\
\hline Age groups & & & & & & 0.16 \\
\hline Under 50 & 689 & 620 & 57.5 & 69 & 51.1 & \\
\hline 50 and over & 525 & 459 & 42.5 & 66 & 48.9 & \\
\hline Marital union & & & & & & 0.288 \\
\hline Yes & 237 & 210 & 66.7 & 27 & 58.7 & \\
\hline Not & 124 & 105 & 33.3 & 19 & 41.3 & \\
\hline
\end{tabular}

n: number of subjects; n': number of subjects in the modalities $\backslash$

Table 1: Socio-demographic characteristics of subjects enrolled

Table 2 compares some clinical and paraclinical characteristics in both groups, as well as the frequency of comorbidities and the clinical stage at admission.

\begin{tabular}{|c|c|c|c|c|c|c|}
\hline \multirow[b]{3}{*}{ Variables $(n=1,235)$} & \multicolumn{5}{|c|}{ Chloroquine + Azithromycin } & \multirow{3}{*}{ p-value } \\
\hline & \multirow[b]{2}{*}{$\mathrm{n}$} & \multicolumn{2}{|c|}{$\begin{array}{l}\text { Yes } \\
(\mathrm{n}=1,100)\end{array}$} & \multicolumn{2}{|c|}{$\begin{array}{l}\text { No } \\
(n=1325)\end{array}$} & \\
\hline & & $\mathrm{n}^{\prime}$ & $\%$ & $\mathrm{n}^{\prime}$ & $\%$ & \\
\hline Pregnant Uterus & & & & & & $0.999^{f}$ \\
\hline Yes & 14 & 13 & 1.5 & 1 & 0.8 & \\
\hline Not & 972 & 852 & 98.5 & 120 & 99.2 & \\
\hline \multicolumn{7}{|l|}{ Comorbidities } \\
\hline HTA (yes) & 276 & 239 & 24.9 & 38 & 29.3 & 0.276 \\
\hline Diabetes (yes) & 151 & 126 & 13.2 & 24 & 18.8 & 0.079 \\
\hline Obesity (yes) & 51 & 41 & 4.3 & 10 & 7.6 & 0.098 \\
\hline Asthma (yes) & 29 & 23 & 2.4 & 6 & 4.8 & 0.125 \\
\hline Heart disease (yes) & 41 & 34 & 3.6 & 7 & 5.3 & 0.324 \\
\hline HIV (yes) & 12 & 12 & 1.3 & 0 & 0.0 & $0.389^{f}$ \\
\hline TBC (yes) & 19 & 18 & 1.9 & 1 & 0.8 & $0.714_{\mathrm{f}}$ \\
\hline Chronic nephropathy (yes) & 11 & 11 & 1.1 & 0 & 0.0 & $0.609^{\mathrm{f}}$ \\
\hline Clinical classification at admission & & & & & & 0.003 \\
\hline Severe/critical stage & 258 & 211 & 22.2 & 47 & 35.3 & \\
\hline Moderate stage & 160 & 145 & 15.2 & 15 & 11.3 & \\
\hline Mild stage & 667 & 596 & 62.6 & 71 & 53.4 & \\
\hline Pathological results at the ECG & & & & & & 0.503 \\
\hline Yes & 65 & 50 & 76.9 & 15 & 88.2 & \\
\hline Not & 17 & 15 & 23.1 & 2 & 11.8 & \\
\hline
\end{tabular}

Table 2: Distribution of comorbidities, clinical and paraclinical characteristics of patients at admission 
With no number of subjects; No number of subjects in the modalities; fisher's exact test.

Except for the clinical stage at admission, the distribution of comorbidities and other clinical and paraclinical data included in this table is not significantly different between the two groups.
The histograms illustrating the distribution of patient propensity scores in Figure 1 shows, in the CT group, a score ranging from 6.5 to 1 and in the second group from 5 to nearly 1 . Matching is therefore visually possible with a very large majority of subjects.

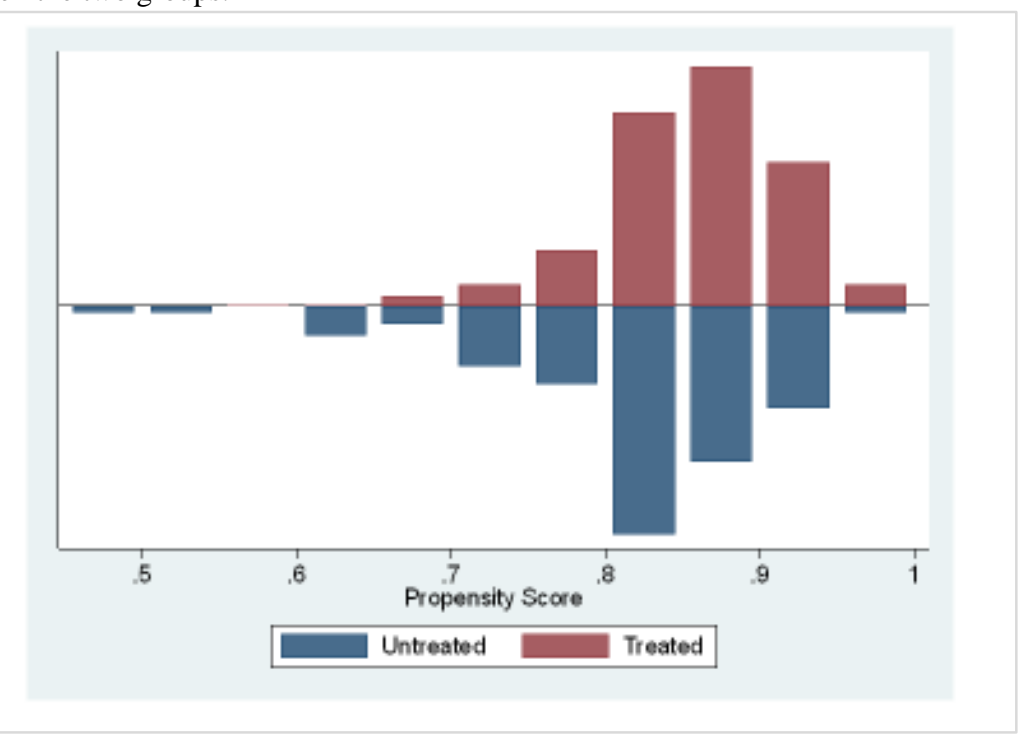

Figure 1: Distribution of histograms of Covid19 patient propensity scores matching by treatment received

\section{Effects of TC on clinical condition and lethality}

\begin{tabular}{|l|l|}
\hline $\begin{array}{l}\text { Adjusted \% difference (IC95\%) asymptomatic patients } \\
\text { on J10 }\end{array}$ & Adjusted p-value \\
\hline $11.5 \%(3.6$ to $19.6 \%)$ & $\mathbf{0 . 0 0 5}$ \\
\hline $\begin{array}{l}\text { With CI - Confidence Interval } \\
*=\text { propensity-score matching, robust standard errors }\end{array}$ \\
\hline
\end{tabular}

Table 3: Patient status at hospitalization D10, adjusted to propensity score

After adjusting the propensity score (matching according to sociodemographic characteristics, comorbidities and clinical stages at admission), table 3 shows a significantly positive outcome in asymptomatic patients on the $10^{\text {th }}$ day of treatment.

\begin{tabular}{|l|l|}
\hline Adjusted difference in lethality (IC95\%) & Adjusted p-value \\
\hline$-7.7 \%(-12.2$ to $3.2 \%)$ & $\mathbf{0 . 0 0 1}$ \\
\hline & \\
\hline $\begin{array}{l}\text { With CI - Confidence Interval } \\
*=\text { propensity-score matching, robust standard errors }\end{array}$ & \\
\hline
\end{tabular}

\section{Table 4: Adjusted effect of TC on lethality by clinical stage}

After adjusting for the propensity score (matching according to sociodemographic characteristics, comorbidities, and clinical stages at admission), table 4 shows that lethality is significantly lower in the presence of CT.

\begin{tabular}{|l|l|l|l|l|l|l|}
\hline & \multicolumn{5}{|l|}{ Chloroquine + Azithromycin } & \\
\hline & \multicolumn{5}{|l|}{$\begin{array}{l}\text { Yes } \\
(\mathbf{n = 0 . 9 5 8 )} \\
\text { (n=0.128) }\end{array}$} & p-value \\
\hline Variable & $\mathbf{n}$ & $\mathbf{n}^{\prime}$ & $\%$ & $\mathbf{n}^{\prime}$ & $\%$ & \\
\hline
\end{tabular}

\section{Adverse events}

Table 5a: Adverse events 


\begin{tabular}{|l|l|l|l|l|l|l|}
\hline Unwanted events & \multicolumn{1}{l|}{} & & & $0.999^{\mathrm{f}}$ \\
\hline Yes & 21 & 19 & 3.2 & 2 & 2.8 & \\
\hline Not & 644 & 574 & 96.8 & 70 & 97.2 & \\
\hline
\end{tabular}

$\mathrm{n}$ : number of subjects; n': number of subjects in the modalities; f: fisher's exact test.

Table 5a reveals that there is no significant difference in the occurrence of adverse events in both groups.

\begin{tabular}{|l|l|l|}
\hline Adverse event & MedDRA Code & SOC (MedDRA) \\
\hline Pruritus & 10037087 & Skin and subcutaneous tissue disorders \\
\hline Rash & 10037844 & Skin and subcutaneous tissue disorders \\
\hline Epigastralgia & 10048944 & Gastrointestinal disorders \\
\hline Constipation & 10017977 & Gastrointestinal disorders \\
\hline Tinging & 10029305 & Skin and subcutaneous tissue disorders \\
\hline Shortness of breath & 10038716 & $\begin{array}{l}\text { Respiratory, Thoracic and Mediastinal } \\
\text { Disorders }\end{array}$ \\
\hline Palpitations & 10082206 & Cardiac disorders \\
\hline Bradycardia & 10007521 & Cardiac disorders \\
\hline
\end{tabular}

Table 5b: Nature of adverse events

Table 5b: shows that adverse effects were generally minor and pruritus-type, digestive disorders, heart palpitations and shortness of breath.

R2. Predictors of patient survival at hospitalization D15

A total of 988 hospitalized patients were followed up to D15.

The 4 figures $(2,3,4$, and 5) below show the predictors that have remained significant in multivariate analysis (Cox model).

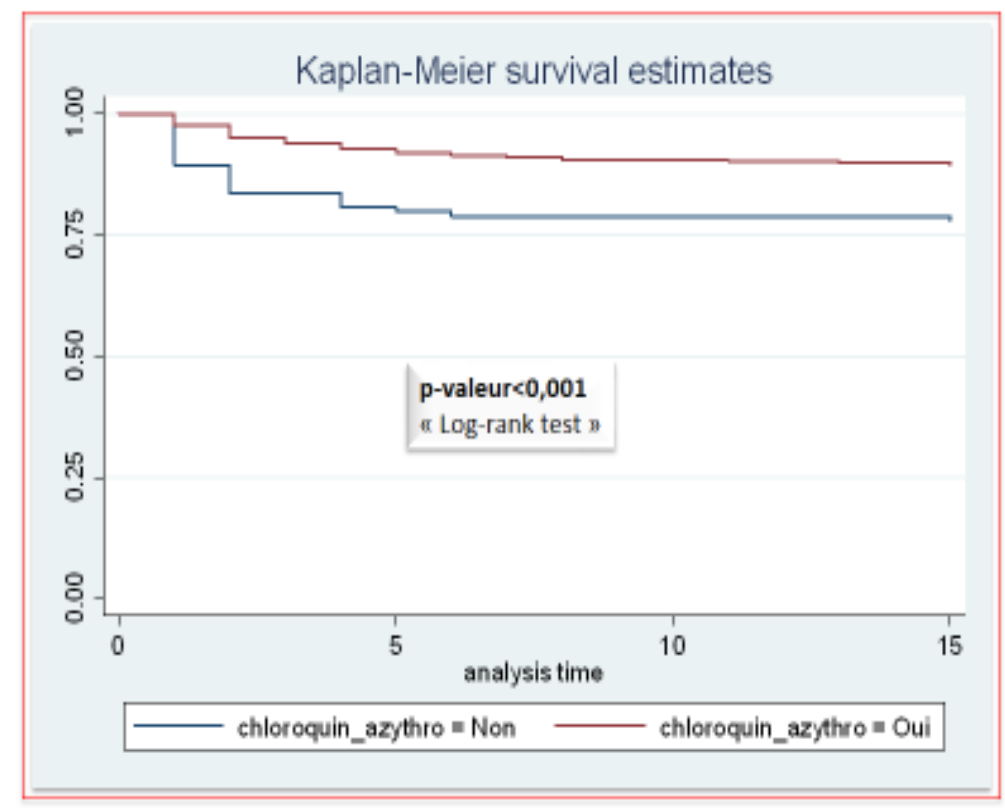

Figure 2: CT Survival Curve Patient survival is significantly better with CT 


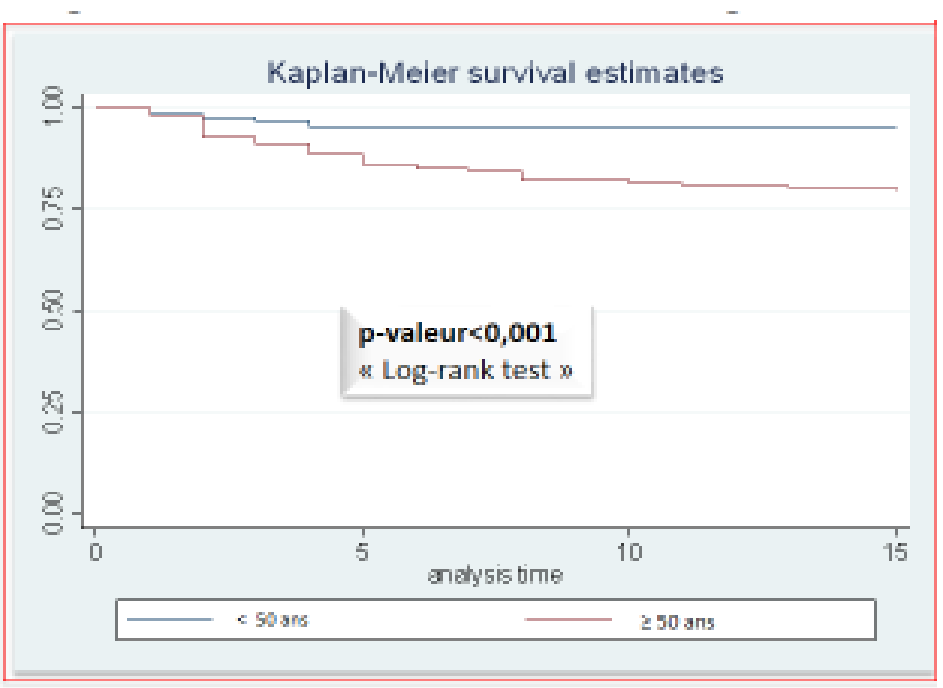

Figures 3: Age-based survival curve Patient survival is significantly better in under-50s

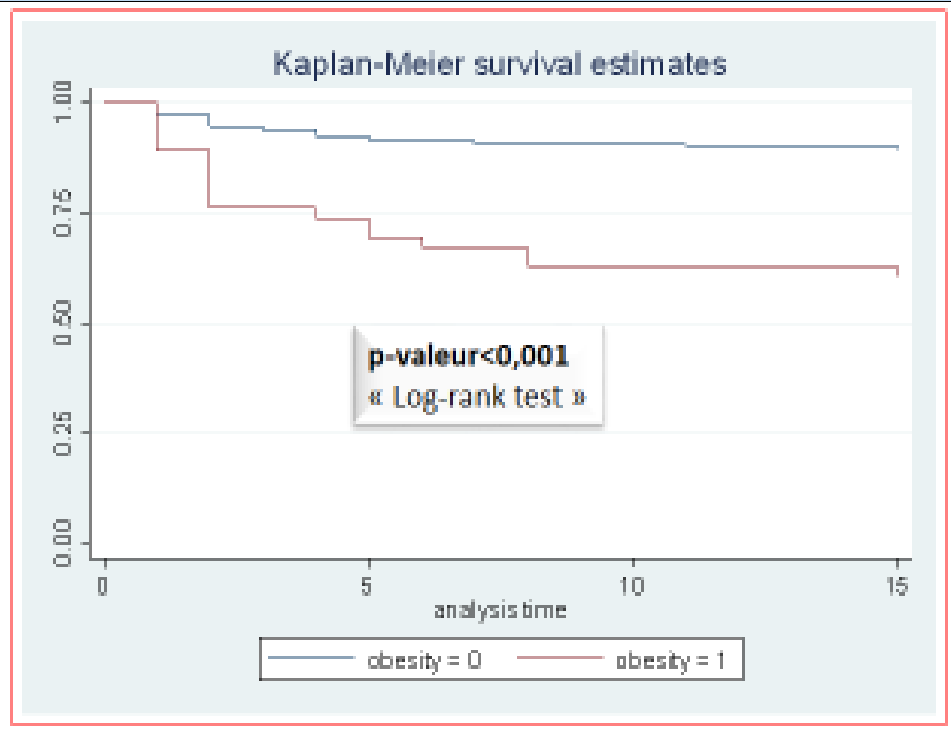

Figure 4: Obesity Survival Curve Patient survival is significantly better in the absence of obesity.

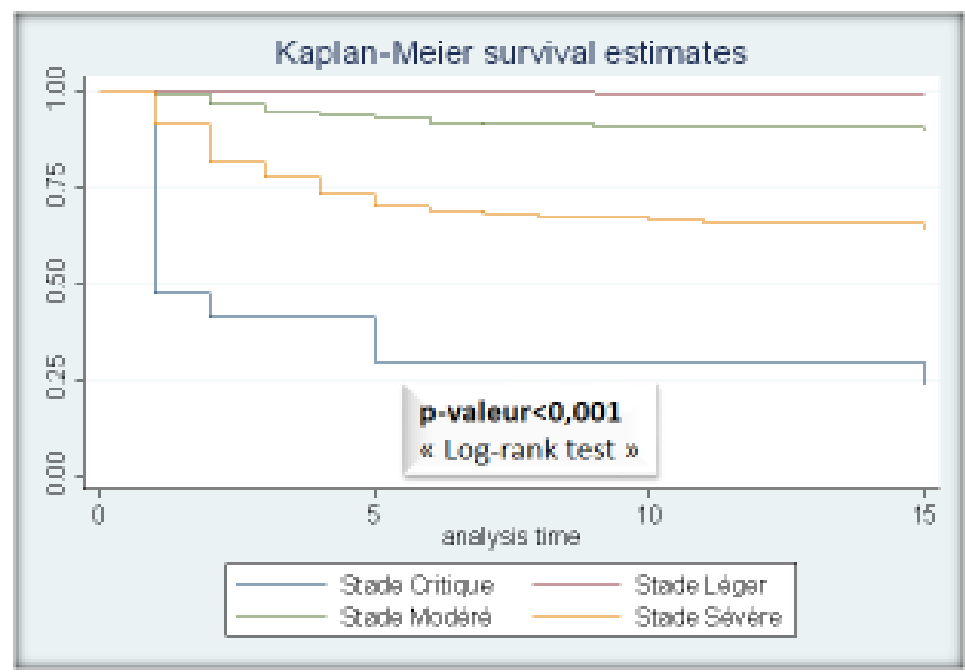

Figure 5: Survival curve depending on the clinical stage. Patient survival is significantly different depending on the clinical stage at admission. It is better in the light stage. 


\begin{tabular}{|l|l|l|}
\hline Independent variables & aHR (IC95\%) & p-value \\
\hline Chloroquine + Azithromycin & & 0.026 \\
\hline Yes & 1 & \\
\hline No & $1.9(1.1$ to 3.3$)$ & \\
\hline Age $\geq 50$ & & 0.003 \\
\hline Yes & $2.3(1.3$ to 3.9$)$ & \\
\hline No & 1 & \\
\hline Obesity & & 0.009 \\
\hline Yes & $2.2(1.2$ to 3.9$)$ & \\
\hline No & 1 & \\
\hline Clinical classification at admission & & $<0.001$ \\
\hline Severe/critical stage & $43.2(16.2$ to 115.7$)$ & \\
\hline Moderate stage & $5.3(1.7$ to 16.4$)$ & \\
\hline Light stadium & 1 & \\
\hline
\end{tabular}

aHR- Adjusted Hazard Ratio

Table 6: Instant risk of death based on independent variables (Cox Model)

After adjustment (table 6), patient survival is significantly associated with treatment administered, age group, obesity, and clinical stage at admission.

Moreover, the instantaneous risk of death is significantly high in patients who have not received conventional treatment, aged 50 and over, obese, and at critical, severe and moderate clinical stages.

\section{Discussion}

The current clinical observation shows essentially a beneficial effect on clinical symptoms and lethality at D10 in COVID-19 patients admitted in the 10 treatment centers at Kinshasa. Main predictors of lethality identified were: age over 50 years and comorbidities including obesity, HTA and diabetes mellitus. Conventional treatment was linked to a positive outcome at D15 in the study group; and no harmful adverse events were recorded in the two groups.

Despite the comparison with a group containing far fewer subjects who did not receive conventional treatment (1,100 subjects vs 135$)$, the use of propensity score allowed to minimize the disparities due to the sample size (Figure 3).

The predominance of male gender among positive subjects for SARS CoV-2 observed in this study is in line with demographic data previously reported by other authors [23-25], and even in a recent study from our country [26]. This gender predominance does not find any obvious explanations at this time. It may be dependent on the protection afforded by the $\mathrm{X}$ chromosome and the sex hormones that could influence innate and adaptive immunity, as reported previously by Long-Quan Li et al [25], and also by Jaillon et al [27]. These authors have suggested the impact of sexual dimorphism on the different susceptibility to infections or autoimmune diseases according to the gender.

The negative impact of comorbidities, including obesity, HTA and diabetes mellitus, on the global mortality is widely reported. It may reflect the prevalence of these pathologies worldwide; with a trend towards male predominance according to WHO declaration [28]. According to the age, our study has found more subjects under the age of 50 years in disagreement with observation from developed countries, where the COVID-19 seems to affect older people [29, 30]. This could be explained by lower life expectancy in developing countries compared to developed countries in Europe, North America, or Asia.

HTA was the main comorbidity in both groups, along with diabetes mellitus and obesity, as in most previous publications [30, 31].

Symptom improvement at D10 was better in patient under conventional treatment ( $86 \%$ vs 63\%); mainly in early administration as observed in an analysis of 126 studies published in October 2020, showing a significantly positive effect on mortality rate, length of hospitalization, and symptom amendment in COVID-19 patient [18].

The positive effect of HCQ was also described by a Chinese team, in a randomized study conducted in Wuhan, China, reporting faster clinical improvement and that of thoracic scannographic images on D6, in 31 out of 62 patients under $400 \mathrm{mg}$ of hydroxychloroquine per day for 5 days [32]. In a systematic review of the literature, Sarma et al showed significant efficacy of HCQ alone or in association on clinical and virological healing, as well as on death or deleterious course of the disease [16].

The predictors of lethality identified in this work were age, clinical stage at admission, and presence of comorbidities. These factors are widely recognized by many authors at present.

Looking at the possible effect of CT on lethality, the current study reveals its slight benefit comparing the two groups. The effects of hydrocholoroquine alone or associated with azithromycin on mortality are far from consensual. However, this observation is consistent with the results presented by Arshad et al in a multicenter retrospective survey conducted in Michigan (USA). These authors described an improvement in survival when hydroxychloroquine, azithromycin, or combination were administered within 2 days of hospitalization [33].

Our study is also enhanced by data from Marseille in France, showing a lower mortality rate among 1,061 patients receiving equivalent doses of the combination HCQ- AZT, as in our CT [30]. In the same trend, Ladapo et al conducted a meta-analysis of randomized studies to determine the preventive effect of early and outpatient HCQ treatment on new infections, hospitalization, and death. These authors concluded that the drug had a positive effect on all three outcomes and reported minor adverse events, especially gastrointestinal disorders [34]. 
However, our results are in disagreement with those from two New York teams, reporting no benefit on clinical outcome and mortality reduction with the same combination therapy (35). The same observation has been described by Geleris et al, showing neither clinical improvement, nor reduction in mortality rate among 1,376 moderate and severe COVID-19 patients in the same state [36].

These disparities could be explained by the difference in the severity of disease and associated comorbidities at hospital admission, the therapeutic protocol applied, the delay in hospital reference, the available equipment and staff expertise. Early treatment is a determining factor in the prognosis of patients treated for COVID-19. Early outpatient administration of HCQ-AZ to symptomatic COVID-19 patients at high risk of hospitalization and rapid worsening has been shown to be beneficial [37].

Unlike the major side effects, especially cardiac ones, described by some authors [36, 37], this study found only minor manifestations, including pruritus and gastrointestinal disorders. Our data are in agreement with other studies, using the same protocols at almost the same drug doses. These results argue for the safety of hydroxychloroquine in this indication, imposing nevertheless an electrocardiographic control beforehand, as well as a monitoring of possible electrolytic disorders.

Few limitations need to be considered in the interpretation of the results presented.

The impact of adjuvant interventions, including oxygen therapy in both groups has not been formally investigated. Recording data in treatment centers that do not have an equivalent level of expertise may have influenced the future of patients. These limitations could have affected the validity of the results of this study, showing the safety of the national protocol in use in our country, as opposed to the major adverse effects described mainly in Western publications.

These encouraging effects on clinical and safety parameters, as reported elsewhere in Sub-Saharan Africa, justify the continuation of this therapeutic protocol at this time.

\section{Conclusion}

This study aimed to add to the existing literature, some evidence on the efficacy and safety of hydroxychloroquine and azithromycin as suggested drugs for COVID-19. The results of this observational clinical study show a beneficial effect of hydroxychloroquine-azithromycin combination on symptoms and lethality in COVD-19 patients. We report the absence of major adverse events that reinforces its widespread use in the global community. The effects of hydroxychloroquine-azythromycin combination on clinical and safety parameters support the continuation of this therapeutic protocol. However, future clinical trials are required to support these findings.

\section{References}

1. Reed Siemieniuk, Jessica Bartoszko, Long Ge, Dena Zeraakar, Ariel Izcovich, Elena Kum et al. (2020) Drug treatments for COVID-19: living systematic review and network metaanalysis. BMJ.

2. Ianevski, A., Andersen, P. I., Merits, A., Bjørås, M., \& Kainov, D. (2019) Expanding the activity spectrum of antiviral agents. Drug Discovery Today, 24(5), 1224-1228.

3. Vincent MJ, Bergeron S, Benjannet S, Erickson BR, Rollin PE, Ksiazek TG, et al. (2005) Chloroquine is a potent inhibitor of SARS coronavirus infection and spread. Virol J. 2:69.

4. Pietro Emanuele Napoli and Matteo Nioi. (2020) Global Spread of Coronavirus Disease 2019 and Malaria: An Epidemiological Paradox in the Early Stage of Pandemic. J Clin Med. 9 (4):1138.
5. Christian Devaux, Jean Marc Rolain, Philippe Colson, Didier Raoult. (2020) New insights on the antiviral effects of chloroquine against coronavirus: what to expect for COVID-19. Int J Antimicrob Agents. 105938.

6. Dan Zhou, Sheng-Ming Dai, Qiang Tong. (2020) COVID-19: a recommendation to examine the effect of hydroxychloroquine in preventing infection and progression. J Antimicrob Chemother. Jul1. 75(7):1667-1670.

7. Liu J, Cao R, Xu M, Wang X, Zhang H, Hu H, et al. (2020) Hydroxychloroquine a less toxic derivative of chloroquine, is effective in inhibiting SARS-CoV-2 infection in vitro. Cell Discov. 6:16.

8. Schrezenmeier E, Dörner T. (2020) Mechanismsof action of hydroxychloroquine and chloroquine: implication for rheumatology. Nat Rev Rheumatol. 16:155-166.

9. Yao X, Ye F, Zhang M, Cui C, Huang B, Niu P, et al. (2020) In vitro antiviral activity and projection of optimized dosing design of hydroxychloroquine for the treatment of severe acute respiratory syndrome coronavirus 2 (SARS-CoV-2). Clin Infect Dis. 71: 732-739.

10. Gautret P, Lagier J, Parola P, Hoang V,Meddeb L, Mailhe M et al. (2020) Hydroxy chloroquine and Azithromycin as a treatment of COVID-19: results of an open-label nonrandomized clinical trial. Int J Antimicrob Agents. 56:105949.

11. Vabret N, Britton Gj, Gruber C , Hegle S,Kim J. Kuksin M, et al. (2020) Immunology of COVID-19: current state of the science. Immunity. 52:910-941.

12. Raja Bhattacharya, Sampurna Chowdhury, Rishav Mukherjee, Anita Nandi, Manish Kulshrestha, Rohini Ghosh, Souvik Saha. Pre exposure hydroxychloroquine prophylaxis for covd-19 in healthcare workers: a retrospective cohort.

13. Sanket Shah, Saibal Das, Avinash Jain, Durga Prasanna Misra, and Vir Singh Negi. (2020) A systematic review of the prophylactic role of chloroquine and hydroxychloroquine in coronavirus disease-19. Int J Rheum Dis. 23 (5): 613-619.

14. Antonella d'Arminio Monforte, Alessandro Tavelli, Francesca Bai, and Giulia Marchetti. (2020) Effectiveness of hydroxychloroquine in COVID-19 disease: A done and dusted deal? Int J Infect Dis. Oct; 99: 75-76.

15. Rosenberg ES, Dufort EM, Udo T Wilberschied LA, Kumar J, Tesoriero J? et al. (2020) Association of treatment with hydroxychloroquine or azithromycin with in-hospital mortality in patients with COVID-19 in New-York State. JAMA. 323: 2493-502.

16. Sarma P, Kaur H, Kumar H, Mahendru D. (2020) Virological and clinical cure in COVID-19 Patients treated with hydroxychloroquine: A systematic Review and Meta-Analysis. J Med Virol.

17. Arshad S, Kilgore P, Chaudhry ZS, Jacobsen G, Wang DD, Huitsing K et al. (2020) Treatment with hydroxychloroquine, Azithromycin , and combination in patients with COVID-19. Int J Infect Dis 97:396-403.

18. HCQ is effective for COVID-19 when used early: analysis of 126 studies. Covid Analysis, October 20, 2020.

19. Grassin-Delyle S, Salvator H, Brollo M, Catherinot E, Sage E, Couderc I-J, et al. (2020) Chloroquine inhibits the release of inflammatory cytokines by human lung explants. Clin Infect Dis.

20. Jang C-H, Choi j-h? Byun M-S, Jue D-M. (2006) Chloroquine inhibits production of TNF- $\alpha$, IL- $1 \beta$ and IL- 6 from lipopolysaccharide-stimulated human monocytes macrophages by different modes. Rheumatology. 45:703-710.

21. Dimitrios Giannis, Ioannis A Ziogas and Panagiota Gianni. (2020) Coagulation disorders in Coronavirus infected patients: 
COVID-19, SARS-CoV-1, MERS-CoV and lessons from the past. J Clin Virol. 127:104362.

22. Jessica E. Nord, Prediman K. Shah, Renee Z Rinaldi, Michael $\mathrm{H}$ Weisman. (2004) Hydroxychloroquine cardiotoxicity in systemic lupus erythematosus: a report of 2 cases and review of the literature. Semin Arthritis Rheum. 33(5):336-351.

23. Catherine Gebhard, Vera Regitz-Zagrosek, Hannelore K Neuhauser, Rosemary Morgan, Sabra L Klein. (2020) Impact of sex and gender on COVID-19 outcomes in Europe. Biol Sex Differ. 11(1):29.

24. Wiersinga WJ, Rhodes A, Cheng AC, Peacook SJ, Prescott HC. (2020) Pathophysiology, transmission, diagnosis, and treatment of coronavirus disease 2019 (COVID-19): a review. JAMA. 324; 782-793.

25. Long-Quan Li, Tian Huang, Yong-Qing Wang, Zheng-Ping Wang, Tao-Bi Huang, Hui-Yun Zhang. (2020) COVID-19 patients, Clinical Characteristics, discharge rate and fatality rate of meta-analysis. J Med Virol. 92(6):577-583.

26. Jean B Nachega, Daniel katuashi Ishoso, John Otshudiema Otokoye, Michel P Hermans, Gisèle Mbuyi, Jean Marie Kayembe et al. (2020) Clinical Characteristics and outcomes of Patients Hospitalized for COVID-19 in Africa: Early Insights from the Democratic Republic of The Congo. Am J Trop Med. $1-10$.

27. Jaillon S, Berthenet K, Garlanda C. (2019) Sexual dimorphism in innate immunity. Clin Rev Allergy Immunol. 56(3):308-321.

28. Boniol M, McIsaac M, Xu L, Wuliji T, Diallo K, Campbell J. (2020) Gender equity in the health workforce: analysis of 104 countries. Geneva: World Health Organization; 2019. WHO Ref. No. WHO/HIS/HWF/Gender/WP1/2019.1.

29. Kui L, Fang YY, Deng Y, et al. Clinical characteristics of novel coronavirusus cases in tertiary hospitals in Hubei Province. Clin Med J.
30. Million M, Lagier JC, Gautret $\mathrm{Ph}$, Colson $\mathrm{Ph}$, Fournier PE, Amrane S et al. (2020) Eearly treatment of COVID-19 patients with hydroxychloroquine and azithromycin: A retrospective analysis of 1061 cases in Marseille, France). Travel Med Infect Dis. 35: 101738.

31. Jing Yang, Ya Zheng, XiGou KePu, Zhaofeng Chen, GingHong Guo et al. (2020) Prevalence of comorbidities and its effects in patients infected with SARS-CoV-2: a systematic review and meta-analysis. Int J Infect Dis. 94: 91-95.

32. Chen Z, Hu J, Zhang Z, Jiang S, Han S, Yan D, Zhuang R, Hu B, Zhang Z. (2020) Efficacy of hydroxychloroquine in patients with COVID-19: results of a randomized clinical trial. MedRxiv and bioRxiv.

33. Arshad S, Kilgore P, Chaudhry ZS, Jacobsen G, Wand dd, Huitsing K, Brar I, Alangaden GJ, et al. (2020) Treatment with hydroxychloroquine, azithromycin, and combination in patients hospitalized with COVID-19. Int.J Infect Dis. 97:396-403.

34. Ladapo JA, McKinnon JE,McCullough PA, Risch H. (2020) Randomized controlled trials of early ambulatory hydroxychloroquine in the prevention of COVID-19 infection,hospitalization, and death : meta-analysis.

35. Rosenberg ES, Dufort EM, Udo T, Wilberrschied LA, Kumar J, Tesoriero J,Weinberg P, Kirkwood J et al. (2020) Association of treatment with hydroxychloroquine orAzithromycin With InHospital Mortality In New York State. JAMA. 323(24); 24932502.

36. Geleris J, Sun Y, Platt J, Zucker J, Baldwin M, Hripcsak G, Labella A et al. (2020) Observational study of hydroxychloroquine in hospitalized patients with COVID-19. N Engl J Med. 382: 2411-2418.

37. Risch HA. (2020) Early outpatient treatment of symptomatic, high-risk COVID-19 patients that should be ramped up immediately as key to the pandemic crisis. Am J Epidemiol. 189(11): 1218-1226.
This work is licensed under Creative Commons Attribution 4.0 License

\section{To Submit Your Article Click Here: Submit Manuscript}

DOI: $10.31579 / 2692-9406 / 065$
Ready to submit your research? Choose Auctores and benefit from:

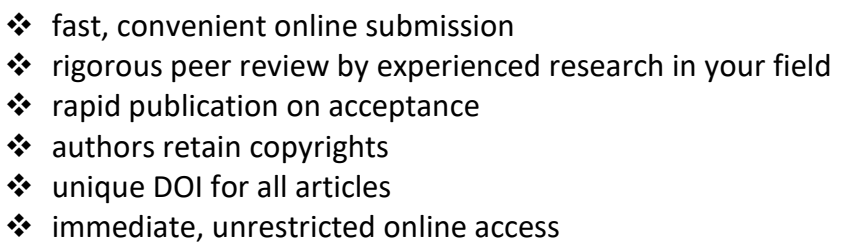

At Auctores, research is always in progress.

Learn more www.auctoresonline.org/journals/biomedical-research-andclinical-reviews- 